\title{
Joint effect of hypertension and lifestyle-related risk factors on the risk of brain microbleeds in healthy individuals
}

\author{
Megumi Hara ${ }^{1}$, Yusuke Yakushiji ${ }^{2}$, Hinako Nannri ${ }^{1}$, Satoshi Sasaki ${ }^{3}$, Tomoyuki Noguchi ${ }^{4}$, \\ Masanori Nishiyama ${ }^{5}$, Tatsumi Hirotsu ${ }^{5}$, Junko Nakajima ${ }^{5}$ and Hideo Hara ${ }^{2}$
}

Brain microbleeds (MBs) are potential risk factors for future stroke, and hypertension is an established risk factor for MBs. However, data on other lifestyle-related risk factors and their joint effects with hypertension are limited. We enrolled 860 adults who underwent 1.5-T brain magnetic resonance imaging and had no history of stroke. Information on clinical risk factors was obtained from health-screening tests, and dietary history was assessed using a validated, brief, self-administered dietary questionnaire. Subjects were divided into three groups (no MBs, deep MBs and lobar MBs), which were compared for the potential risk factors; their joint effects with hypertension were assessed by logistic regression. Biologic interaction was estimated with the synergy index. After adjustment for possible confounders, age and systolic and diastolic blood pressures were found to be associated with the presence of MBs in a dose-dependent manner, especially in the case of deep MBs. With regard to lifestyle-related factors, current smoking status was significantly associated with deep MBs, and the odds ratio was 2.73 (95\% confidence interval $(\mathrm{Cl})$ 1.15-6.48). We found that hypertension and current smoking status, higher alcohol consumption or lower calcium intake had joint effects on the risk of MBs and that hypertension and current smoking status had synergistic additive action (synergy index, 6.30; 95\% $\mathrm{Cl} 1.07-37.13$ ). These results suggest that approaches combining lowering blood pressure and smoking cessation may greatly reduce the risk of MBs and contribute to preventing stroke. Hypertension Research (2013) 36, 789-794; doi:10.1038/hr.2013.26; published online 11 April 2013

Keywords: brain microbleeds; lifestyle; MRI; prevention; risk factors

\section{INTRODUCTION}

Cerebral microbleeds (MBs) observed by gradient-echo $\mathrm{T} 2^{\star}$-weighted MRI are considered biomarkers of two types of pathological changes, according to their location. ${ }^{1}$ The MBs located strictly in the lobar region (lobar MBs) are associated with cerebral amyloid angiopathy, whereas those in deep regions (deep MBs) are indicative of hypertensive vasculopathy. ${ }^{2,3}$ A recent longitudinal study suggested that the presence of MBs can predict hemorrhage and ischemic stroke, even in healthy elderly individuals; ${ }^{4}$ therefore, it is reasonable to expect that risk factors for stroke may be associated with MBs.

Hypertension is strongly associated with $\mathrm{MBs},{ }^{1,5}$ especially deep $\mathrm{MBs}^{2,3}$ while its associations with lifestyle-related risk factors are inconsistent. ${ }^{3,6}$ The Rotterdam scan study showed the association between smoking and deep $\mathrm{MBs}^{3}{ }^{3}$ while the Framingham study did not find a clear association. ${ }^{6}$ With regard to dietary factors, high sodium intake, ${ }^{7-9}$ heavy alcohol consumption, ${ }^{10-12}$ low calcium intake $^{13,14}$ and low animal protein intake ${ }^{15}$ are considered risk factors for stroke; however, the associations of these factors, except alcohol use, with MBs have never been examined. ${ }^{16}$ Only one study reported that heavy alcohol use was an independent predictor of MBs; however, the sample size of that study was quite small $(n=87){ }^{16}$ The present study, therefore, analyzed the relationships between MBs and lifestyle-related risk factors and the joint effects of these factors with hypertension on MBs in the general population without stroke history.

\section{METHODS}

Study design and subjects

The study is based on the Kashima Scan Study, an ongoing population-based cohort study investigating age-related brain changes on MRI. The details of the cohort study have been reported elsewhere. ${ }^{17-19}$ Briefly, it includes individuals who were $\geqslant 30$ years of age, did not have any disability in the instrumental activities of daily living, had the ability to independently make visits for current health-screening tests of the brain, voluntarily provided written informed consent, did not have a history of neurological disorders or brain injuries, and whose complete MRI images were available. Data were collected

${ }^{1}$ Department of Preventive Medicine, Faculty of Medicine, Saga University, Saga, Japan; ${ }^{2}$ Division of Neurology, Department of Internal Medicine, Faculty of Medicine, Saga University, Saga, Japan; ${ }^{3}$ Department of Social and Preventive Epidemiology, School of Public Health, The University of Tokyo, Tokyo, Japan; ${ }^{4}$ Department of Radiology, Faculty of Medicine, Saga University, Saga, Japan and ${ }^{5}$ Yuai-Kai Oda Hospital, Saga, Japan

Correspondence: Dr M Hara, Department of Preventive Medicine, Faculty of Medicine, Saga University, 5-1-1 Nabeshima, Saga 849-8501, Japan.

E-mail: harameg@cc.saga-u.ac.jp

Received 27 November 2012; revised 27 January 2013; accepted 31 January 2013; published online 11 April 2013 
on their clinical characteristics, such as age; gender; years of education; history of stroke and ischemic heart diseases; family history of stroke; smoking status; and presence of hypertension, diabetes mellitus and hyperlipidemia. A total of 1237 consecutive adults underwent health-screening tests of the brain at their own expense between December 2005 and November 2010. They were asked to respond to a validated, brief, self-administered diet history questionnaire (BDHQ) via mail within 1 month of the health-screening tests, and 893 subjects replied $(72.2 \%)$. Compared with the non-responding subjects, those who responded included a higher proportion of women, had a higher mean age, and had a lower proportion of current smokers; however, both groups were similar in terms of the number of subjects with MBs. After excluding 33 subjects who had a history of stroke $(n=7)$ or ischemic heart disease ( $n=26), 860$ subjects (411 men, 449 women; age, 33-84 years) were included in this study. This study has been approved by the human ethics review board of Saga University.

\section{Dietary assessment}

Dietary history was assessed using the BDHQ. ${ }^{20}$ Responses to the BDHQ were checked for completeness and, where necessary, clarified by telephonic conversations with the subjects. The BDHQ is a four-page, fixed-portion questionnaire that enquires about the consumption frequency of selected foods, to estimate the dietary intake of 58 food and beverage items during the preceding month. The BDHQ consists of five sections: (1) intake frequency of food and nonalcoholic beverage items, (2) daily intake of rice and miso soup, (3) frequency of drinking and amount per drink for alcoholic beverages, (4) usual cooking methods and (5) general dietary behavior. The validity of the BDHQ has been reported elsewhere. ${ }^{20}$ Pearson correlation coefficients with dietary record for energy-adjusted intakes of alcohol, sodium, calcium and animal protein were $0.92,0.60,0.77$ and 0.62 for men and $0.87,0.61,0.67$ and 0.49 for women, respectively; thus, they had reasonable ranking ability.

\section{Cardiovascular risk assessment}

Hypertension was defined as systolic blood pressure of $>140 \mathrm{~mm} \mathrm{Hg}$ and/or diastolic blood pressure of $>90 \mathrm{~mm} \mathrm{Hg}$ or use of antihypertensive medication. Diabetes mellitus was defined as a fasting serum glucose level of $\geqslant 126 \mathrm{mg} \mathrm{dl}^{-1}$, hemoglobin Alc levels of $\geqslant 6.9 \%$ (National Glycohemoglobin Standardization Program (NGSP)), or use of anti-diabetic medication. Dyslipidemia was defined as fasting serum total cholesterol levels of $\geqslant 220 \mathrm{mg} \mathrm{dl}^{-1}$ and/or fasting serum triglyceride levels of $\geqslant 200 \mathrm{mg} \mathrm{dl}^{-1}$ and/ or the use of antihyperlipidemic agents. Patients who were smokers at the time of analysis were classified as current smokers. Information regarding the duration of education was collected for each subject.

\section{Brain MRI}

MRI was performed using a 1.5-T scanner (EXCELART Vantage, version 7.0; Toshiba Medical Systems, Tokyo, Japan). Gradient-echo T2*-weighted MRI, axial T1-weighted imaging, fluid-attenuated inversion recovery imaging, and fast spine-echo T2-weighted imaging were performed using the same section thickness, matrix and parameters described previously. ${ }^{17,18}$

\section{Rating of brain MBs}

MBs were defined on gradient-echo MRI as rounded areas of signal loss, $\leqslant 10 \mathrm{~mm}$ in diameter. Two investigators who were blinded to subject data reviewed the number and location of MBs. Symmetrical hypointensities in the globus pallidus caused by calcification and flow void artifacts of pial vessels were carefully excluded. Subjects were categorized into three groups: without MBs, lobar MBs (cerebral cortices, subcortical white matter, or periventricular white matter) and deep MBs (basal ganglia; thalamus; white matter of the corpus callosum; internal, external or extreme capsule; infratentorial structures; brain stem; or cerebellum). The diffuse MBs located in both lobar and deep lesions were considered 'deep' as diffuse MBs are believed to represent chronic extensions of deep MBs. ${ }^{18}$ The inter- and intra-rater reliability for rating MBs, expressed as Cohen's kappa, was 0.73 and 0.86 , respectively, indicating good agreement.

\section{Statistical analysis}

To compare the baseline characteristics and MRI findings between the two groups, the $\chi^{2}$-test and $t$-test were used, as appropriate. We considered separate categories for 'strictly lobar MBs' and 'deep MBs.' The associations of lifestylerelated risk factors with the presence of MBs were examined using a multiple logistic regression model, and the odds ratios and confidence intervals (CIs) of MBs for each risk factor were computed. Selection of potential confounders was based on a priori consideration of their association with MBs. Variables included in the multivariate model were age; sex; BMI; histories of hypertension, diabetes and dyslipidemia; duration of education; current smoking status; alcohol consumption; and intake of sodium, calcium, and animal protein. The joint effects of hypertension and lifestyle-related risk factors were examined. Interaction on the multiplicative scale was assessed by comparing multiplicative models by using the log-likelihood ratio test. Interaction on the additive scale was assessed by calculating the synergy index $(S)$, using the algorithm of Andersson et al. ${ }^{21} S$ was calculated as $\left[\mathrm{RR}_{11}-1\right] /$ $\left[\left(R_{10}-1\right)+\left(R_{01}-1\right)\right]$, where $R_{11}$ is the relative risk for both risk factors present, $\mathrm{RR}_{10}$ is the relative risk for the first risk factor being present and the second risk factor being absent, and $\mathrm{RR}_{01}$ is the relative risk for the first risk factor being absent and the second risk factor being present. $S \neq 1$ indicates the presence of additive interaction. Statistical analysis was performed using the Statistical Analysis System version 9.2 (SAS Institute Japan Ltd, Tokyo, Japan) and Microsoft Excel (version 2010, Microsoft Japan, Tokyo, Japan). Values of $P<0.05$ were considered statistically significant.

\section{RESULTS}

MBs were detected in the brains of 71 of the 860 subjects $(8.3 \%)$. Of these 71 subjects, 28 (3.3\%) had only supratentorial MBs, $33(3.8 \%)$ showed only infratentorial MBs and $10(1.2 \%)$ exhibited MBs in both areas. Thus, the numbers of all MBs, strictly lobar MBs and deep MBs were 71, 28 and 43, respectively. Compared with the subjects without

Table 1 Characteristics of subjects with and without MBs

\begin{tabular}{|c|c|c|c|}
\hline & $\begin{array}{l}\text { Without MBs } \\
(\mathrm{N}=789)\end{array}$ & $\begin{array}{l}\text { With MBs } \\
(\mathrm{N}=71)\end{array}$ & $\mathrm{P}$-value \\
\hline Age (years), mean (s.d.) & $58.5(9.3)$ & $63.1(7.9)$ & $<0.001$ \\
\hline Men, no. (\%) & $340(43.1)$ & $38(53.5)$ & 0.090 \\
\hline Obesity $\left(\mathrm{BMI} \geqslant 25 \mathrm{~kg} \mathrm{~m}^{-2}\right)$, no. (\%) & $191(24.1)$ & $22(30.1)$ & 0.251 \\
\hline SBP (mm Hg), mean (s.d.) & $125.1(18.1)$ & $135.1(16.1)$ & $<0.001$ \\
\hline DBP (mm Hg), mean (s.d.) & $76.4(11.2)$ & $81.8(9.8)$ & $<0.001$ \\
\hline FBG $\left(\mathrm{mg} \mathrm{dl}^{-1}\right)$, mean (s.d.) & $99.5(17.3)$ & $103.4(19.3)$ & 0.088 \\
\hline HbA1c (NGSP) (\%) & $5.57(0.61)$ & $5.59(0.56)$ & 0.749 \\
\hline Triglyceride (mg dl ${ }^{-1}$ ) & $109.9(62.3)$ & $120.7(64.4)$ & 0.166 \\
\hline LDL-C (mg dl $\left.{ }^{-1}\right)$ & $129.5(32.4)$ & $125.3(33.5)$ & 0.299 \\
\hline $\mathrm{HDL}-\mathrm{C}\left(\mathrm{mg} \mathrm{dl}^{-1}\right)$ & $63(16.6)$ & $63.5(16.6)$ & 0.802 \\
\hline $\mathrm{GRF}<60, n(\%)$ & $49(6.2)$ & $6(8.5)$ & 0.46 \\
\hline Antihypertensive drug use, $n(\%)$ & $150(19.0)$ & $34(47.9)$ & $<0.001$ \\
\hline Antihyperlipidemic drug use, $n(\%)$ & $33(4.2)$ & $6(8.5)$ & 0.098 \\
\hline Anti-hyperglycemic drug use, $n(\%)$ & $71(9.0)$ & $10(14.1)$ & 0.160 \\
\hline Hypertension, no. (\%) & $261(33.1)$ & $47(66.2)$ & $<0.001$ \\
\hline Dyslipidemia, no. (\%) & $396(50.2)$ & $47(66.2)$ & 0.01 \\
\hline Diabetes mellitus, no. (\%) & $59(7.5)$ & $10(13.7)$ & 0.059 \\
\hline Current smoking status, no. (\%) & $101(12.8)$ & $14(19.7)$ & 0.101 \\
\hline Alcohol intake (g per day), mean (s.d.) & $10.5(18.2)$ & $14.4(19.9)$ & 0.087 \\
\hline Sodium intake (mg per day) & $4278.1(1345.9)$ & 4414 & 0.416 \\
\hline Calcium intake (mg per day) & $552.4(233.8)$ & $601.8(296.5)$ & 0.176 \\
\hline Animal protein (g per day) & $37.6(18.5)$ & $40.7(25.1)$ & 0.317 \\
\hline Education (years), mean (s.d.) & $12.4(2.3)$ & $12.1(2.6)$ & 0.284 \\
\hline
\end{tabular}

Abbreviations: BMI, body mass index; DBP, diastolic blood pressure; FBG, fasting blood glucose; GFR, glomerular filtration rate; HDL-C, high-density lipoprotein cholesterol; HT, hypertension; LDL-C, low-density lipoprotein cholesterol; MBs, microbleeds; NGSP, Nationa hypertension; LDL-C, low-density lipoprotein cholesterol; MBs, microbleeds; a $P$-values for $\chi^{2}$-test or $t$-test. 


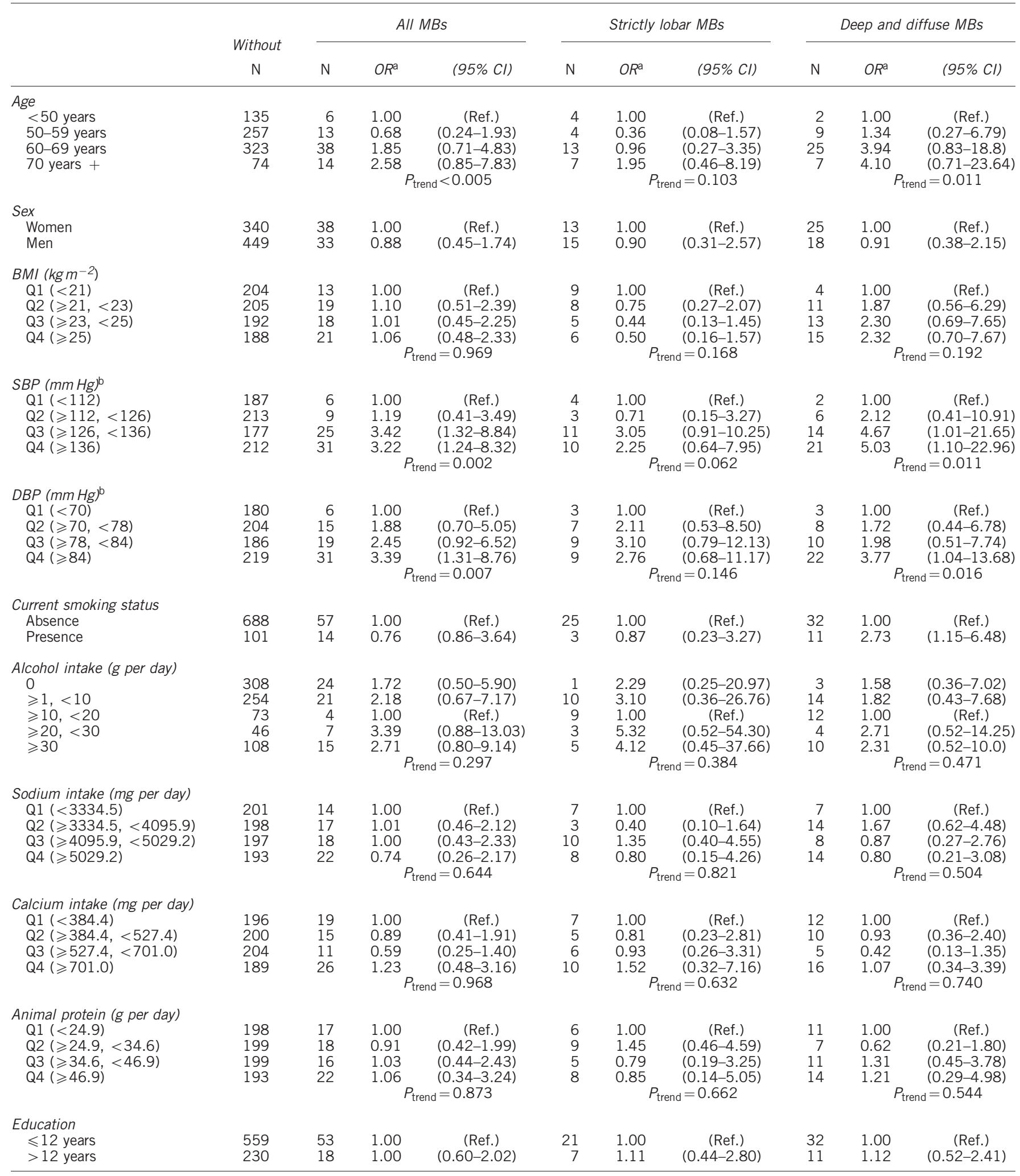

Abbreviations: BMI, body mass index; Cl, confidence interval; DBP, diastolic blood pressure; MBs, microbleeds; OR, odds ratio; ref., reference; SBP, systolic blood pressure.

aAdjusted for age (continuous), sex, BMI (continuous), history of hypertension (yes, no), history of diabetes (yes or no), history of dyslipidemia (yes or no), duration of education (contimuous), current smoking (yes or no), alcohol consumption (contimuous), sodium intake (continuous), calcium intake (continuous) and animal fat intake (continuous).

${ }^{\mathrm{b}}$ Adjusted for above variables, except for history of hypertension (yes, no). 
Table 3 Characteristics of subjects with and without hypertension

\begin{tabular}{|c|c|c|c|}
\hline & $\begin{array}{l}\text { Without-HT } \\
(\mathrm{N}=552)\end{array}$ & $\begin{array}{c}\text { With-HT } \\
(\mathrm{N}=308)\end{array}$ & P-value \\
\hline Age, years, mean (s.d.) & $57.3(9.3)$ & $61.9(8.4)$ & $<0.001$ \\
\hline Men, no. $(\%)$ & $214(38.8)$ & $164(53.2)$ & $<0.001$ \\
\hline Obesity (BMI $\geqslant 25 \mathrm{~kg} \mathrm{~m}^{-2}$ ), no. (\%) & $102(18.5)$ & $107(34.7)$ & $<0.001$ \\
\hline SBP (mm Hg), mean (s.d.) & $117.1(12.8)$ & $141.6(15.7)$ & $<0.001$ \\
\hline DBP (mm Hg), mean (s.d.) & $72.5(9.2)$ & $84.6(10.1)$ & $<0.001$ \\
\hline FBG $\left(\mathrm{mg} \mathrm{dl}^{-1}\right)$, mean (s.d.) & $97.6(16.7)$ & $103.8(18.2)$ & $<0.001$ \\
\hline HbAlc (NGSP) (\%) & $5.52(0.63)$ & $5.65(0.56)$ & 0.005 \\
\hline Triglyceride ( $\mathrm{mg} \mathrm{dl}^{-1}$ ) & $103.7(57.7)$ & $123.5(68.6)$ & $<0.001$ \\
\hline LDL-C $\left(\mathrm{mg} \mathrm{dl}^{-1}\right)$ & $130.1(29.1)$ & $127.4(37.8)$ & 0.270 \\
\hline HDL-C (mg dl-1) & $63.8(15.8)$ & $61.7(18.0)$ & 0.093 \\
\hline $\mathrm{GRF}<60, n(\%)$ & $28(5.1)$ & $27(8.8)$ & 0.034 \\
\hline Antihypertensive drug use, $n$ (\%) & $0(0)$ & $184(59.7)$ & $<0.001$ \\
\hline Antihyperlipidemic drug use, $n(\%)$ & $38(6.9)$ & $43(14.0)$ & $<0.001$ \\
\hline Anti-hyperglycemic drug use, $n(\%)$ & $19(3.4)$ & $20(6.5)$ & 0.039 \\
\hline Dyslipidemia, no. (\%) & $264(47.8)$ & $179(58.1)$ & 0.004 \\
\hline Diabetes mellitus, no. (\%) & $33(6.0)$ & $36(11.7)$ & 0.003 \\
\hline Current smoking status, no. (\%) & $71(12.9)$ & $44(14.3)$ & 0.557 \\
\hline Alcohol intake (g per day), mean (s.d.) & $8.8(17.1)$ & $14.5(19.8)$ & $<0.001$ \\
\hline Sodium intake (mg per day) & $\begin{array}{c}4178.0 \\
(1345.2)\end{array}$ & $\begin{array}{c}4488.7 \\
(1331.3)\end{array}$ & 0.001 \\
\hline Calcium intake (mg per day) & $545.4(235.9)$ & $576.5(245.6)$ & 0.068 \\
\hline Animal protein (g per day) & $36.8(18.3)$ & 39.9 (20.3) & 0.025 \\
\hline Education, years, mean (s.d.) & $12.6(2.3)$ & $12.0(2.5)$ & $<0.001$ \\
\hline
\end{tabular}

Abbreviations: BMI, body mass index; DBP, diastolic blood pressure; FBG, fasting blood glucose; GFR, glomerular filtration rate; HbAlc, hemoglobin Alc; HDL-C, high-density lipoprotein cholesterol; HT, hypertension; LDL-C, low-density lipoprotein cholesterol; NGSP, National Glycohemoglobin Standardization Program; SBP, systolic blood pressure. a $P$-values for $\chi^{2}$-test or $t$-test.
MBs, those with MBs were of significantly higher age, had higher systolic and diastolic blood pressure, and had a higher prevalence of hypertension or dyslipidemia; however, the two groups were similar with regard to the other lifestyle-related factors (Table 1).

The prevalence of MBs in subjects aged $<50$ years, 50-59 years, 60-69 years, and $\geqslant 70$ years was $4.3 \%, 4.8 \%, 10.5 \%$ and $15.9 \%$, respectively, thereby showing an increase with age (Table 2). The odds ratio of MBs increased with age, systolic and diastolic blood pressure and current smoking status, especially in the case of deep MBs (Table 2). No significant associations were found between sex, BMI, duration of education or dietary risk factors and the presence of MBs (Table 2) although these factors were significantly associated with hypertension (Table 3 ).

The joint effects of current smoking status and hypertension tended to be stronger than the sum of the independent effects of each factor for all MBs and deep MBs, and the synergy index for this combination was statistically significant: 6.30 (95\% CI 1.07-37.13) for all MBs and 5.02 (95\% CI 1.02-24.75) for deep MBs (Table 4). The joint effects of hypertension and high alcohol intake or low calcium intake were observed, although the synergy index for these combinations was not significant (Table 4). The other lifestyle-related factors had no joint effects with hypertension (data not shown).

\section{DISCUSSION}

A recent longitudinal study suggested that the presence of MBs can be used to predict the occurrence of hemorrhage and ischemic stroke; ${ }^{4}$ thus, the risk factors for stroke were expected to be associated with MBs. The present cross-sectional study indicated that the presence of MBs was significantly associated with higher age and blood pressure, as reported previously, ${ }^{2,3,6}$ and that age, blood pressure and current

Table 4 Joint effect of lifestyle-related risk factors and hypertension on microbleeds

\begin{tabular}{|c|c|c|c|c|c|c|c|c|c|c|c|}
\hline \multirow[b]{2}{*}{ Hypertension } & \multirow{2}{*}{$\begin{array}{l}\text { Lifestyle-related } \\
\text { Risk factors }\end{array}$} & \multirow{2}{*}{$\begin{array}{c}\text { Without MB } \\
\text { No. }\end{array}$} & \multicolumn{3}{|c|}{ All MBs } & \multicolumn{3}{|c|}{ Strictly lobar MBs } & \multicolumn{3}{|c|}{ Deep MBs } \\
\hline & & & No. & $O R^{a}$ & $(95 \% \mathrm{Cl})$ & No. & $O R^{a}$ & $(95 \%$ Cl) & No. & $O R^{a}$ & $(95 \% \mathrm{Cl})$ \\
\hline \multicolumn{12}{|c|}{ Current smoking } \\
\hline$(-)$ & $(-)$ & 459 & 22 & 1.00 & (Ref.) & 12 & 1.00 & (Ref.) & 10 & 1.00 & (Ref.) \\
\hline$(-)$ & $(+)$ & 69 & 2 & 0.58 & $(0.13-2.65)$ & 0 & NA & & 2 & 1.24 & $(0.25-6.22)$ \\
\hline$(+)$ & $(-)$ & 229 & 35 & 2.39 & $(1.30-4.38)$ & 13 & 2.13 & $(0.89-5.13)$ & 22 & 2.72 & $(1.20-6.18)$ \\
\hline \multirow[t]{2}{*}{$(+)$} & $(+)$ & 32 & 12 & 7.13 & $(2.86-17.76)$ & 3 & 3.94 & $(0.88-17.65)$ & 9 & 10.84 & $(3.53-33.29)$ \\
\hline & & \multicolumn{2}{|c|}{ Synergy index } & 6.30 & (1.07-37.13) & & NA & & & 5.02 & $(1.02-24.75)$ \\
\hline \multicolumn{12}{|l|}{ High alcoholb } \\
\hline$(-)$ & $(-)$ & 469 & 22 & 1.00 & (Ref.) & 11 & 1.00 & (Ref.) & 11 & 1.00 & (Ref.) \\
\hline$(-)$ & $(+)$ & 59 & 2 & 0.75 & $(0.16-3.44)$ & 1 & 0.95 & $(0.11-8.27)$ & 1 & 0.66 & $(0.08-5.50)$ \\
\hline$(+)$ & $(-)$ & 212 & 34 & 2.78 & $(1.52-5.08)$ & 12 & 2.46 & $(0.99-6.09)$ & 22 & 3.14 & $(1.42-6.93)$ \\
\hline \multirow[t]{2}{*}{$(+)$} & $(+)$ & 49 & 13 & 4.51 & $(1.92-10.59)$ & 4 & 4.30 & $(1.10-16.81)$ & 9 & 4.97 & $(1.75-14.17)$ \\
\hline & & \multicolumn{2}{|c|}{ Synergy index } & 2.31 & $(0.58-9.21)$ & & 2.34 & $(0.23-23.59)$ & & 2.22 & $(0.45-10.93)$ \\
\hline \multicolumn{12}{|l|}{ Low calciumc } \\
\hline$(-)$ & $(-)$ & 395 & 19 & 1.00 & (Ref.) & 9 & 1.00 & (Ref.) & 10 & 1.00 & (Ref.) \\
\hline$(-)$ & $(+)$ & 133 & 5 & 0.86 & $(0.30-2.57)$ & 3 & 1.14 & $(0.27-4.88)$ & 2 & 0.66 & $(0.13-3.31)$ \\
\hline$(+)$ & $(-)$ & 198 & 33 & 2.67 & $(1.42-5.01)$ & 12 & 2.67 & $(1.04-6.85)$ & 21 & 2.79 & $(1.23-6.34)$ \\
\hline \multirow[t]{2}{*}{$(+)$} & $(+)$ & 63 & 14 & 4.13 & $(1.76-9.67)$ & 4 & 3.08 & $(0.77-12.30)$ & 10 & 4.95 & $(1.71-14.30)$ \\
\hline & & \multicolumn{2}{|c|}{ Synergy index } & 2.02 & $(0.57-7.22)$ & & 1.15 & $(0.15-8.74)$ & & 2.73 & $(0.53-14.11)$ \\
\hline
\end{tabular}

Abbreviations: $\mathrm{Cl}$, confidence interval; MBs, microbleeds; NA, not analyzed; OR, odds ratio; Ref., reference.

${ }^{a}$ Adjusted for age (continuous), sex, BMI (continuous), history of diabetes (yes or no), history of dyslipidemia (yes or no), duration of education (contimuous), current smoking (yes or no), alcohol consumption (contimuous), sodium intake (continuous), calcium intake (continuous) and animal fat intake (continuous).

consumption (contimuous), sodium intake (continuous), calcium intake
bDaily ethanol intake $\geqslant 30 \mathrm{~g}$ per day is defined as high alcohol intake.

${ }^{c} \mathrm{Q} 4$ (the highest quartile) is defined as high intake, and Q1 (the lowest quartile) is defined as low intake. 
smoking status were associated with deep MBs. The most important findings of the study are that the joint effects of hypertension and current smoking status, higher alcohol consumption or lower calcium intake on the risk of MBs, as well as the significant synergistic additive interaction for the combination of hypertension and current smoking status. These associations were clear for MBs in deep lesions, which are considered indicative of hypertensive microangiopathy. ${ }^{2,3}$

Several studies and pooled analyses have provided evidence of a joint effect of blood pressure and smoking status on the risk of stroke. ${ }^{2-24}$ With regard to MBs, the Rotterdam scan study revealed the associations between MBs and hypertension or smoking separately, but the interactions between them was not examined. ${ }^{3}$ To the best of our knowledge, this is the first study showing the significant joint effect of smoking and hypertension on MBs. Similarly, the existence of the joint effects of stroke and MBs might be plausible because the presence of MBs is considered an imaging biomarker of future hemorrhage and ischemic stroke. ${ }^{4}$ The synergistic interaction between current smoking status and hypertension on $\mathrm{MB}$ risk suggest that these risk factors together increase the possibility of the development of $\mathrm{MBs}$, although the exact pathophysiological explanation remains unclear. Both hypertension and smoking are closely associated with endothelial dysfunction, ${ }^{25}$ cerebral small vessel disease or arteriosclerosis ${ }^{26}$ due to increased oxidative stress and inflammation. ${ }^{27}$ Thus, smoking may accelerate the effect of hypertension on $\mathrm{MB}$ risk.

Joint effects of hypertension and heavy drinking or low calcium intake on $\mathrm{MB}$ risk were also suspected, although statistically significant additive interactions were absent. Multivariate analysis showed that both hypertension and heavy alcohol use are significant predictors of MBs. ${ }^{16}$ However, their joint effects have never been examined. Heavy alcohol drinking not only raises blood pressure through the stimulation of the sympathetic nervous system but also aggravates coagulation disorders. ${ }^{10}$ We speculate that heavy alcohol drinking may enhance the microvascular damage due to hypertension and increase the risk of MBs by reduced coagulability. The association between calcium intake and $\mathrm{MB}$, as well as the joint effect of hypertension and calcium intake on $\mathrm{MBs}$, has not been reported although several cohort studies have shown that daily calcium intake reduces stroke risk. ${ }^{13,14}$ Umesawa et al. ${ }^{14}$ mentioned that the hypotensive effect of dietary calcium helps explains its protective effect against hemorrhagic stroke and that the potential effect of dietary calcium on reduced platelet aggregation and total cholesterol level may reduce the risk of ischemic stroke. As MBs are considered biomarkers of future hemorrhage and ischemic stroke, ${ }^{4}$ it is provable that the combination of hypertension and low calcium intake may have a synergistic effect on MB risk.

In the present study, we did not find any associations of MBs with intakes of sodium and animal fat, and duration of education, which are considered risk factors for stroke. ${ }^{7-15}$ Contrarily, they were significantly associated with hypertension. Therefore, we speculate that these factors may increase the risk of hypertension and may indirectly influence $\mathrm{MB}$ risk.

The present study has several limitations. First, the definition of hypertension was too simple; we did not have data on home measurements of blood pressure although home blood pressure measurements are considered important to diagnose masked hypertension, which is associated with cardiovascular events. ${ }^{28}$ As we used only one-point measurement of blood pressure to define hypertension, subjects with masked hypertension might have been included in the group without hypertension, while those with whitecoat hypertension might have been included in the hypertension group. This misclassification would have influenced the nature of the associations determined in this study. Second, a selection bias might have been present. The study subjects comprised those who provided information regarding their dietary habits by mail within 1 month of their health-screening tests (response rate was 72.2\%). Responders might be healthier than non-responders in this study; however, the presence of MBs did not differ among them. Thus, this selection bias may cause reserve association, due to the reduced distribution of baseline characteristics among them. Third, there may be intrinsic information bias in our assessment of lifestyle-related factors, including dietary factors. However, the Pearson correlation coefficients for dietary records showed fairly good reasonable ranking ability. ${ }^{20}$ If any misclassification occurred, it may be nondifferential with respect to MBs and possibly lead to the underestimation of the true associations. Fourth is the lack of information on medications, such as antithrombotic drug use or statin use. A previous study has shown the association between antithrombotic drug use and MBs. ${ }^{1,29}$ We excluded subjects with a history of stroke and ischemic heart diseases, who might use antithrombotic drugs. With regard to cholesterol level and MBs, epidemiological studies have reported an increased risk of hemorrhagic stroke in populations with low cholesterol levels, ${ }^{30-32}$ and one study reported an association of hemorrhagic stroke and statin use, ${ }^{33}$ although this association was not verified in a metaanalysis of randomized-controlled trials of statin therapy. ${ }^{34}$ The lack of information about antihyperlipidemic drugs, especially statins, may have been responsible for the fact that we did not find any association between MBs and low-density lipoprotein-cholesterol level in this study. Fifth, the small sample size in this study may have led to the failure in detecting significant additive interactions between hypertension and heavy alcohol drinking or low calcium intake. Finally, residual confounding might be present, although we did adjust for potential confounding factors in multivariate analysis.

In conclusion, we found that hypertension and current smoking status, higher alcohol consumption and lower calcium intake exert joint effects on $\mathrm{MB}$ risk, with hypertension being a predominant risk factor for MBs. Smoking and hypertension had a statistically significant synergistic risk of MBs. These findings suggest that combining blood-pressure-lowering approaches and smoking cessation may greatly reduce the risk of $\mathrm{MBs}$ and contribute to preventing stroke.

\section{CONFLICT OF INTEREST}

The authors declare no conflict of interest.

\section{ACKNOWLEDGEMENTS}

We wish to thank Ms Miwako Koga, Ms Mitsuko Uematsu, Mr Koji Miyazaki, Ms Mai Miyamoto, and the medical staff of Yuai-Kai Oda Hospital for their help with medical interviews, acquisition of imaging data and data entry.

1 Greenberg SM, Vernooij MW, Cordonnier C, Viswanathan A, AI-Shahi Salman R, Warach S, Launer LJ, Van Buchem MA, Breteler MM. Cerebral microbleeds: a guide to detection and interpretation. Lancet Neurol 2009; 8: 165-174.

2 Vernooij MW, van der Lugt A, Ikram MA, Wielopolski PA, Niessen WJ, Hofman A, Krestin GP, Breteler MM. Prevalence and risk factors of cerebral microbleeds: the rotterdam scan study. Neurology 2008; 70: 1208-1214.

3 Poels MM, Vernooij MW, Ikram MA, Hofman A, Krestin GP, van der Lugt A, Breteler MM. Prevalence and risk factors of cerebral microbleeds: an update of the rotterdam scan study. Stroke 2010; 41: S103-S106.

4 Bokura H, Saika R, Yamaguchi T, Nagai A, Oguro H, Kobayashi S, Yamaguchi S. Microbleeds are associated with subsequent hemorrhagic and ischemic stroke in healthy elderly individuals. Stroke 2011; 42: 1867-1871. 
5 Poels MM, Ikram MA, van der Lugt A, Hofman A, Krestin GP, Breteler MM, Vernooij MW. Incidence of cerebral microbleeds in the general population: the rotterdam scan study. Stroke 2011; 42: 656-661.

6 Jeerakathil T, Wolf PA, Beiser A, Massaro J, Seshadri S, D'Agostino RB, DeCarli C. Stroke risk profile predicts white matter hyperintensity volume: the framingham study. Stroke 2004; 35: 1857-1861.

7 Nagata C, Takatsuka N, Shimizu N, Shimizu H. Sodium intake and risk of death from stroke in japanese men and women. Stroke 2004; 35: 1543-1547.

8 Umesawa M, Iso H, Date C, Yamamoto A, Toyoshima H, Watanabe Y, Kikuchi S, Koizumi A, Kondo T, Inaba Y, Tanabe N, Tamakoshi A. Relations between dietary sodium and potassium intakes and mortality from cardiovascular disease: The japan collaborative cohort study for evaluation of cancer risks. Am J Clin Nutr 2008; 88 195-202.

9 Strazzullo P, D'Elia L, Kandala NB, Cappuccio FP. Salt intake, stroke, and cardiovascular disease: Meta-analysis of prospective studies. BMJ 2009; 339: b4567.

10 Reynolds K, Lewis B, Nolen JD, Kinney GL, Sathya B, He J. Alcohol consumption and risk of stroke: a meta-analysis. JAMA 2003; 289: 579-588.

11 Iso H, Baba S, Mannami T, Sasaki S, Okada K, Konishi M, Tsugane S. Alcohol consumption and risk of stroke among middle-aged men: The jphc study cohort $\mathrm{i}$. Stroke 2004; 35: 1124-1129.

12 Patra J, Taylor B, Irving H, Roerecke M, Baliunas D, Mohapatra S, Rehm J. Alcohol consumption and the risk of morbidity and mortality for different stroke types-a systematic review and meta-analysis. BMC Public Health 2010; 10: 258.

13 Umesawa M, Iso H, Date C, Yamamoto A, Toyoshima H, Watanabe Y, Kikuchi S, Koizumi A, Kondo T, Inaba Y, Tanabe N, Tamakoshi A. Dietary intake of calcium in relation to mortality from cardiovascular disease: the jacc study. Stroke 2006; 37: 20-26.

14 Umesawa M, Iso H, Ishihara J, Saito I, Kokubo Y, Inoue M, Tsugane S. Dietary calcium intake and risks of stroke, its subtypes, and coronary heart disease in japanese: the jphc study cohort i. Stroke 2008; 39: 2449-2456.

15 Iso $\mathrm{H}$, Sato S, Kitamura A, Naito Y, Shimamoto T, Komachi Y. Fat and protein intakes and risk of intraparenchymal hemorrhage among middle-aged japanese. Am J Epidemiol 2003; 157: 32-39.

16 Copenhaver BR, Hsia AW, Merino JG, Burgess RE, Fifi JT, Davis L, Warach S, Kidwell CS. Racial differences in microbleed prevalence in primary intracerebral hemorrhage. Neurology 2008; 71: 1176-1182.

17 Yakushiji Y, Nishiyama M, Yakushiji S, Hirotsu T, Uchino A, Nakajima J, Eriguchi M, Nanri Y, Hara M, Horikawa E, Kuroda Y. Brain microbleeds and global cognitive function in adults without neurological disorder. Stroke 2008; 39: 3323-3328.

18 Yakushiji Y, Noguchi T, Hara M, Nishihara M, Eriguchi M, Nanri Y, Nishiyama M, Hirotsu T, Nakajima J, Kuroda Y, Hara H. Distributional impact of brain microbleeds on global cognitive function in adults without neurological disorder. Stroke 2012; 43 1800-1805.

19 Yakushiji Y, Nanri Y, Hirotsu T, Nishihara M, Hara M, Nakajima J, Eriguchi M, Nishiyama M, Hara H, Node K. Marked cerebral atrophy is correlated with kidney dysfunction in nondisabled adults. Hypertens Res 2010; 33: 1232-1237.
20 Kobayashi S, Honda S, Murakami K, Sasaki S, Okubo H, Hirota N, Notsu A, Fukui M Date $C$. Both comprehensive and brief self-administered diet history questionnaires satisfactorily rank nutrient intakes in japanese adults. J Epidemiol 2012; 22: 151-159.

21 Andersson T, Alfredsson L, Kallberg H, Zdravkovic S, Ahlbom A. Calculating measures of biological interaction. Eur J Epidemiol 2005; 20: 575-579.

22 Ohgren B, Weinehall L, Stegmayr B, Boman K, Hallmans G, Wall S. What else adds to hypertension in predicting stroke? An incident case-referent study. J Intern Med 2000; 248: 475-482.

23 Yamagishi $\mathrm{K}$, Iso $\mathrm{H}$, Kitamura A, Sankai $T$, Tanigawa $T$, Naito $\mathrm{Y}$, Sato $\mathrm{S}$, Imano $\mathrm{H}$, Ohira T, Shimamoto T. Smoking raises the risk of total and ischemic strokes in hypertensive men. Hypertens Res 2003; 26: 209-217.

24 Nakamura K, Barzi F, Lam TH, Huxley R, Feigin VL, Ueshima H, Woo J, Gu D, Ohkubo T, Lawes CM, Suh I, Woodward M. Cigarette smoking, systolic blood pressure, and cardiovascular diseases in the Asia-Pacific region. Stroke 2008; 39: 1694-1702.

25 Endemann DH, Schiffrin EL. Endothelial dysfunction. J Am Soc Nephrol 2004; 15 1983-1992.

26 Iso H. Lifestyle and cardiovascular disease in japan. J Atheroscler Thromb 2011; 18 $83-88$.

27 Sierra C, Coca A, Schiffrin EL. Vascular mechanisms in the pathogenesis of stroke. Curr Hypertens Rep 2011; 13: 200-207.

28 Shimada K, Fujita T, Ito S, Naritomi H, Ogihara T, Shimamoto K, Tanaka H, Yoshiike N The importance of home blood pressure measurement for preventing stroke and cardiovascular disease in hypertensive patients: a sub-analysis of the japan hypertension evaluation with angiotensin ii antagonist losartan therapy (j-health) study, a prospective nationwide observational study. Hypertens Res 2008; 31 1903-1911.

29 Vernooij MW, Haag MD, van der Lugt A, Hofman A, Krestin GP, Stricker BH, Bretele MM. Use of antithrombotic drugs and the presence of cerebral microbleeds: the rotterdam scan study. Arch Neurol 2009; 66: 714-720.

30 Iso H, Jacobs DR Jr, Wentworth D, Neaton JD, Cohen JD. Serum cholesterol levels and six-year mortality from stroke in 350,977 men screened for the multiple risk factor intervention trial. N Engl J Med 1989; 320: 904-910.

31 Zhang X, Patel A, Horibe H, Wu Z, Barzi F, Rodgers A, MacMahon S, Woodward M. Cholesterol, coronary heart disease, and stroke in the asia pacific region. Int $J$ Epidemiol 2003; 32: 563-572.

32 Sturgeon JD, Folsom AR, Longstreth WT Jr, Shahar E, Rosamond WD, Cushman M. Risk factors for intracerebral hemorrhage in a pooled prospective study. Stroke 2007; 38: 2718-2725.

33 Amarenco P, Bogousslavsky J, Callahan A 3rd, Goldstein LB, Hennerici M, Rudolph AE, Sillesen H, Simunovic L, Szarek M, Welch KM, Zivin JA. High-dose atorvastatin after stroke or transient ischemic attack. N Engl J Med 2006; 355: 549-559.

34 McKinney JS, Kostis WJ. Statin therapy and the risk of intracerebral hemorrhage: a meta-analysis of 31 randomized controlled trials. Stroke 2012; 43 2149-2156. 